\title{
Freqüência de indivíduos com intolerância à glicose em jejum em um hospital universitário: comparação de critérios diagnósticos
}

\author{
Frequency of individuals with impaired fasting glucose in a university hospital: \\ comparison of diagnostic criteria
}

Dilênia de Oliveira Cipriano'; Heidy Vieira Lima²; Ana Aparecida dos Santos Souza²; Maria Cleide Freire Clementino da Silva ${ }^{3}$; Ana Célia Oliveira dos Santos ${ }^{4}$

\begin{abstract}
unitermos resumo
Intolerância à glicose de jejum $\quad$ A intolerância à glicose de jejum (IG)), uma condição metabólica que na maioria das vezes precede o Critérios de diagnóstico diabetes, geralmente não causa sintomas, necessitando da dosagem da glicemia de jejum para ser diagnosticada. Em 2003, o Comitê de Especialistas em Diagnóstico e Classificação do Diabetes Mellitus (Expert Committee on the Diagnosis and Classification of Diabetes Mellitus) recomendou a redução do ponto de corte para o diagnóstico da IG] de $\geq 110 \mathrm{mg} / \mathrm{dl}$ para $\geq 100 \mathrm{mg} / \mathrm{dl}$, endossado pela Sociedade Brasileira de Diabetes (SBD), em 2005. No entanto ainda não há consenso sobre a adoção desses valores. $O$ objetivo deste estudo foi avaliar a freqüência de pacientes com IG) atendidos, de agosto a novembro de 2005, no Hospital Universitário Oswaldo Cruz da Universidade de Pernambuco (HUOC/UPE), comparando os critérios de diagnóstico. Foram avaliadas glicemias de 428 indivíduos, sendo $65 \%$ do sexo feminino. A média de idade dos pacientes foi de 52,3 anos $( \pm 15,4)$, sendo que $78,3 \%$ desses estavam na faixa etária $\geq 40$ anos. Do total da amostra, $4 \%$ tiveram glicemia de jejum sugestiva de diabetes ( $\geq 126 \mathrm{mg} / \mathrm{dl}$ ), enquanto $7 \%$ dos resultados foram compatíveis com IG), segundo os critérios anteriormente adotados $(\geq 110 \mathrm{mg} / \mathrm{dl} \mathrm{e}$ $<126 \mathrm{mg} / \mathrm{dl}$ ), e esses pacientes tinham idade $\geq 40$ anos. Porém, utilizando os novos critérios de diagnóstico ( $\geq 100 \mathrm{mg} / \mathrm{dl}$ e $<126 \mathrm{mg} / \mathrm{dl}$ ), a incidência de indivíduos com IG) passou para $14,7 \%$ e a faixa etária dos indivíduos acometidos para $\geq 20$ anos. Esses resultados indicam maior sensibilidade na detecção de pacientes com IG], após redefinição dos critérios de diagnóstico, nas faixas etárias avaliadas.
\end{abstract}

abstract

Impaired fasting glucose (IFG), a condition that most times precedes diabetes, generally does not produce any symptoms. In 2003, the Expert Committee on the Diagnosis and Classification of Diabetes Mellitus recommended a lower cutoff point (from $\geq 110 \mathrm{mg} / \mathrm{dl}$ to $\geq 100 \mathrm{mg} / \mathrm{dl}$ ) for fasting plasma glucose to confirm its diagnosis, a measure officially endorsed by the Brazilian Society of Diabetes (BSD) in 2005. The objective of this study was to evaluate the frequency of patients who presented IFG seen at the State University Hospital Oswaldo Cruz, utilizing both criteria of diagnosis, from August to November 2005. The sample consisted of 428 individuals, $65 \%$ of whom were females, with a mean age of 52.3 years $( \pm 15.4)$ and a percentage of $78.3 \%$ of this population were $\geq 40$ years old. From the total sample, $4 \%$ presented a fasting glucose level suggestive of diabetes $(\geq 126 \mathrm{mg} / \mathrm{dl})$, while $7 \%$ presented glycemia values between $\geq 110 \mathrm{mg} / \mathrm{dl}$ and $<126 \mathrm{mg} / \mathrm{dl}$, all of these patients were aged $\geq 40$ years. The new criteria proposed for the diagnosis of IFG showed that the frequency of IFG patients has increased to $14.7 \%$ and their age has fallen to $\geq 20$. These results suggest, with this new diagnosis criterion, a higher sensibility in the detection of IFG among subjects in the age range studied.

\section{key words}

Impaired fasting glucose

Criteria of diagnosis

Diabetes mellitus

1. Mestre em Bioquímica; biomédica do Laboratório Central do Hospital Universitário Oswaldo Cruz da Universidade de Pernambuco (HUOC/UPE).

2. Pós-graduação em Análises Clínicas da Faculdade Frassinetti do Recife (FAFIRE).

3. Pós-graduanda em Patologia Clínica; biomédica do Laboratório Central do HUOC/UPE.

4. Doutora em Ciências Biológicas; professora do Instituto de Ciências Biológicas (ICB) da UPE.

Trabalho realizado no Laboratório Central do HUOC/UPE. 


\section{Introdução}

As transições demográfica, nutricional e epidemiológica ocorridas no século passado determinaram um perfil de risco em que doenças crônicas, como diabetes e hipertensão, assumiram ônus crescente e preocupante. Ambas são condições prevalentes e importantes problemas de saúde pública em todos os países, independentemente de seu grau de desenvolvimento ${ }^{(20)}$.

O diabetes mellitus (DM) é uma disfunção metabólica de múltipla etiologia, caracterizada por hiperglicemia crônica resultante de deficiência na secreção de insulina, de ação da insulina ou de ambos. Estimou-se, em 1995, que 4\% da população adulta mundial era portadora de DM e a projeção, para 2025, é que 5,4\% da população será afetada pelo diabetes, o que equivalerá a aproximadamente 300 milhões de diabéticos. A maior parte desse aumento se dará em países em desenvolvimento, e neles se acentuará o atual padrão de concentração de casos na faixa etária de 45-64 anos ${ }^{(10)}$.

$O$ aumento da incidência de diabetes em termos mundiais tem sido relacionado às modificações de estilo de vida e do meio ambiente decorrentes da industrialização. Essas modificações levam à obesidade, ao sedentarismo e ao consumo de alimentos ricos em calorias e gorduras ${ }^{(12,21)}$.

Em 1997, o Comitê de Especialistas em Diagnóstico e Classificação do Diabetes Mellitus (Expert Committee on the Diagnosis and Classification of Diabetes Mellitus) ${ }^{(17)}$ definiu intolerância à glicose de jejum (IG]) como a faixa entre o limite superior de normalidade $(\geq 110 \mathrm{mg} / \mathrm{dl})$ e o limite inferior de glicemia para o diagnóstico do diabetes $(<126 \mathrm{mg} / \mathrm{dl})$, preceito referendado pela Sociedade Brasileira de Diabetes $(\mathrm{SBD})^{(7)}$. Em 2003, o mesmo comitê recomendou a redução do ponto de corte inferior da glicose para diagnóstico da IG], de $110 \mathrm{mg} / \mathrm{dl}$ para $100 \mathrm{mg} / \mathrm{dl}^{(18)}$, também referendado pela $S B D$, segundo o Consenso Brasileiro sobre Diabetes ${ }^{(16)}$.

Após essas modificações, indivíduos saudáveis passaram a ter diagnóstico de IG], ou pré-diabetes, condição que, na maioria das vezes, precede o diabetes e geralmente não causa sintomas. Existem evidências de que mesmo antes de a doença se manifestar por completo, tanto nas alterações da glicemia como na sintomatologia característica, já ocorrem lesões no sistema circulatório dos indivíduos pré-diabéticos ${ }^{(1)}$. Então, conhecendo o maior risco de desenvolver diabetes, tanto o médico quanto o paciente podem se empenhar no tratamento do pré-diabetes, tomando medidas preventivas para que essa condição não evolua, com medidas de baixo custo, geralmente através de dieta adequada e exercícios físicos ${ }^{(6,16)}$.
Os benefícios clínicos advindos da redução do cut off para a intolerância à glicose de jejum ainda são discutidos ${ }^{(2,}$ 4). A Organização Mundial da Saúde (OMS) ainda não assumiu esses novos valores como recomendação, mantendo aqueles previamente definidos ${ }^{(21)}$.

Ao considerar que o laboratório de análises desempenha um papel fundamental no acompanhamento glicêmico, o objetivo deste estudo foi avaliar a freqüência de pacientes com IG] atendidos no Hospital Universitário Oswaldo Cruz da Universidade de Pernambuco (HUOC/UPE), comparando a freqüência da intolerância à glicose de acordo com os critérios diagnósticos utilizados.

\section{Material e métodos}

Foi realizado um estudo descritivo de corte transversal através de levantamento retrospectivo de pacientes provenientes do Sistema Único de Saúde (SUS), atendidos no laboratório de análises do HUOC/UPE, no período de agosto a novembro de 2005, num momento de redefinição dos critérios de diagnóstico de IG] nesse hospital.

A amostra foi constituída de pacientes ambulatoriais, de ambos os sexos, selecionados a partir da consulta aleatória de solicitação médica de dosagem da glicemia de jejum. Para inclusão no estudo, os indivíduos selecionados deveriam preencher os seguintes critérios: ter idade igual ou superior a 20 anos; não ter diabetes preexistente e/ou histórico de intolerância à glicose. lá em relação às pacientes selecionadas, essas não poderiam estar grávidas. As informações foram retiradas das requisições médicas.

A glicose sérica foi determinada utilizando o analisador Roche/Hitachi 912. Os valores de referência aplicados para identificar a IG] foram:

- SBD (2002): glicemia $\geq 110 \mathrm{mg} / \mathrm{dl} \mathrm{e}<126 \mathrm{mg} / \mathrm{dl}$;

- SBD (2005): glicemia $\geq 100 \mathrm{mg} / \mathrm{dl} \mathrm{e}<126 \mathrm{mg} / \mathrm{dl}$.

Este estudo foi aprovado para estudo pelo Comitê de Ética em Pesquisa com Seres Humanos do Centro Integrado de Saúde Amaury de Medeiros (CEPH/CISAM) da UPE, sob o no $90 / 05$.

\section{Resultados}

Foram avaliados 428 indivíduos, sendo $65 \%$ do sexo feminino. A idade média foi de 52,3 anos $( \pm 15,4)$ : o paciente com a menor idade tinha 20 anos e o com a maior, 83. No estudo, observou-se incidência de $78,3 \%$ para a faixa etária $\geq 40$ anos. 
Do total da amostra, 4\% apresentaram glicemia de jejum sugestiva de diabetes $(\geq 126 \mathrm{mg} / \mathrm{dl})$, enquanto $7 \%$ dos resultados foram compatíveis com IG], segundo os critérios anteriormente adotados ( $\geq 110 \mathrm{mg} / \mathrm{dl} \mathrm{e}<126 \mathrm{mg} / \mathrm{dl}$ ), e todos foram observados em indivíduos com idade $\geq 40$ anos. Porém, utilizando os novos critérios diagnósticos (glicose $\geq$ $100 \mathrm{mg} / \mathrm{dl}$ e $<126 \mathrm{mg} / \mathrm{dl}$ ), a freqüência de indivíduos com IG) passou para $14,7 \%$ e a faixa etária para $\geq 20$ anos, sendo $4,8 \%$ desses com idade inferior a 40 anos.

Ao comparar as freqüências entre os indivíduos diagnosticados com IG], segundo os critérios definidos em $2002^{(7)} \mathrm{e}$ 2005 pela SBD ${ }^{(16)}$, observa-se nitidamente que o novo ponto de corte para a IG] aumenta consideravelmente o número de pacientes diagnosticados como pré-diabéticos e que a distribuição de freqüência das várias faixas de idade entre os dois grupos apresenta o mesmo comportamento, ou seja, aumenta com a idade, porém a faixa etária de indivíduos acometidos passou de $\geq 40$ para $\geq 20$ anos (Tabela).

\section{Discussão}

O diabetes mellitus tipo 2 (DM2) é uma das principais causas de mortalidade no mundo. Apesar da alta prevalência de DM2 em países desenvolvidos, no futuro os países em desenvolvimento serão os mais acometidos, e neles vive a maioria da população mundial(14).

As complicações em decorrência da evolução do diabetes, especificamente naqueles que permanecem anos com alterações na tolerância à glicose, mas sem diagnóstico $\mathrm{e}$ sem o devido tratamento, tornam claras a importância do diagnóstico precoce e a necessidade da implementação do tratamento adequado, com mudanças nos hábitos alimentares e no estilo de vida o mais cedo possível. Sem falar na possibilidade de se tentar evitar a evolução do intolerante à glicose para o DM propriamente dito $(5,9,10)$.

Além dos custos financeiros, o DM acarreta também outros custos associados, como dor, ansiedade, inconveniência e menor qualidade de vida para os doentes e seus familiares. O diabetes apresenta também carga adicional à sociedade, em decorrência da perda de produtividade no trabalho, da aposentadoria precoce e da mortalidade prematura ${ }^{(1)}$.

O diagnóstico correto e precoce do DM e das alterações da tolerância à glicose é extremamente importante, para que se possa tentar com o tratamento, também iniciado precocemente, diminuir a morbimortalidade dessa doença tão freqüente e crescente na população mundial(13).

A utilização da glicemia de jejum - como método de screening para o diagnóstico de diabetes ou [G] - tem demonstrado ser importante na detecção de indivíduos que deveriam ser investigados, pois as taxas de $4 \%$ de glicemia sugestiva para DM e de 7\% de intolerância à glicemia de jejum, utilizando os critérios adotados pela SBD em 2002, por si só mostram o valor do método de seleção empregado.

Um estudo multicêntrico brasileiro realizado em 1992 por Malerbi e Franco ${ }^{(11)}$, encontrou uma freqüência de 7,6\% de diabetes e valores semelhantes de intolerância à glicose $(7,8 \%)$. Esses achados podem refletir o impacto dos fatores ambientais e do estilo de vida, porém a idade é o fator que determina o maior risco para alterações na homeostase da glicose, como observado por Malerbi e Franco(11), Cruz-Filho et al. ${ }^{(3)}$, Torquato et al. ${ }^{(19)}$, e Schaan et al. ${ }^{(15)}$, corroborando este trabalho, bem como o conhecimento prévio de que a tolerância à glicose vai diminuindo com o envelhecimento dos indivíduos.

Portanto, utilizando como ponto de corte as recomendações da SBD (2005) ${ }^{(16)}$, a freqüência de novos casos de
Tabela 1

\begin{tabular}{cccccccc} 
Tabela 1 & \multicolumn{7}{c}{ diagnósticos SBD (2002) e SBD (2005), agrupados quanto à idade } \\
\hline $\begin{array}{c}\text { Faixa etária } \\
\text { (anos) }\end{array}$ & \multicolumn{2}{c}{ Amostra } & \multicolumn{2}{c}{ SBD (2002) } & \multicolumn{2}{c}{ SBD (2005) } & Aumento \\
\hline $20-29$ & 33 & 7,7 & 0 & 0 & 1 & 3 & em vezes (X) \\
$30-39$ & 60 & 14 & 0 & 0 & 2 & 3,3 & 1 \\
$40-49$ & 92 & 21,5 & 3 & 3,3 & 11 & 12 & 3,1 \\
$50-59$ & 94 & 22 & 8 & 8,5 & 15 & 16 & 1,9 \\
$\geq 60$ & 149 & 34,8 & 19 & 12,8 & 34 & 22,8 & 1,8 \\
Total & 428 & 100 & 30 & 7 & 63 & 14,7 & 2,1 \\
\hline
\end{tabular}
diagnósticos SBD (2002) e SBD (2005), agrupados quanto à idade

*IG): Intolerância à glicose de jejum.

Frequiência de IGJ em pacientes atendidos em hospital universitário, utilizando os critérios 
intolerância à glicose de jejum foi bem maior, passando de $7 \%$ para $14,7 \%$ (aumento de 2,1 vezes), sendo o maior aumento registrado na faixa etária entre 20 e 49 anos, o que elevou o número de adultos jovens classificados como portadores de IG]. Resultados semelhantes foram encontrados por Davidson et al.(4), que encontraram aumento de cerca de cinco vezes na ocorrência de IGJ para a idade entre 20 e 50 anos, e de Borch-Johnsen et al. (2004) (2), que demonstraram o aumento da prevalência de IG] em um estudo envolvendo as populações da Dinamarca, França, Índia e EUA.

A faixa etária de indivíduos acometidos abaixou de $\geq 40$ anos de idade para $\geq 20$. Esse dado é importante para se repensar sobre o que preconiza o Consenso Brasileiro sobre Diabetes da SBD (2002)(16), ao indicar o rastreamento do diabetes em todo indivíduo com mais de 45 anos de idade a cada três anos, pois, em se tratando de doença de progressão inexorável, cujas complicações podem ser minimizadas com controle intensivo da glicemia, a determinação cada vez mais precoce do diagnóstico é altamente desejável(8).

Portanto, considerando o aumento da freqüência dos indivíduos classificados como portadores de IG], quando se utilizaram as recomendações de novos critérios de diagnóstico da SBD (2005) $)^{(16)}$, e considerando que os mesmos estavam assintomáticos e saudáveis, bem como atentando para a conhecida associação de hiperglicemia crônica com o desenvolvimento de lesões micro e macrovasculares em longo prazo, fica clara a importância de os laboratórios de análises clínicas se manterem constantemente atualizados quanto a novos critérios diagnósticos dos estados de intolerância à glicose. No entanto, ainda se discute o risco do desenvolvimento de DM em indivíduos com IG] identificados por esse novo cut-off, principalmente considerando as possíveis descriminações trabalhistas para a população de menor faixa etária e os custos para o sistema de saúde com o acompanhamento desses indivíduos ${ }^{(4)}$.

Os dados deste trabalho são insuficientes para informar sobre os benefícios em longo prazo para os portadores de IG) com o uso desses novos parâmetros. Apenas um estudo longitudinal será capaz de responder se esses indivíduos serão sabidamente expostos a um risco maior de desenvolver DM e/ou doença cardiovascular.

\section{Conclusão}

Esses resultados indicam maior sensibilidade na detecção de pacientes com IG] após redefinição dos critérios de diagnóstico, em todas as faixas etárias avaliadas, inclusive em pessoas com menos de 40 anos de idade.

\section{Referências}

I. BARZILAV, J. et al. Cardiovascular disease in older adults with glucose disorders: comparisons of American Diabetes Association of diabetes mellitus with WHO criteria. Lancet, v. 354, p. 622-5, 1999

2. BORCH-JOHNSEN, K. et al. Creating a pandemic of prediabetes: the proposed new diagnostic criteria for impaired fasting glycaemia. Diabetologia, v. 47, n. 8, p. 1396-402, 2004.

3. CRUZ-FILHO, R.A. et al. Papel da glicemia capilar de jejum no diagnóstico precoce da diabetes mellitus: correlação com fatores de risco cardiovascular. Arq Bras Endocrinol Metab, v. 46, n. 3, p. 255-9, 2002

4.DAVIDSON,M.B.; LANDSMAN, P.B.;ALEXANDER, C.M. Lowering the criterion for impaired fasting glucose will not provide clinical benefit. Diabetes Care, v. 26, n. I2, p. 3329-30, 2003.

5. ENGELGAU, M.M.; NARAYAN, K.M.V.; HERMAN,W.H. Screening for type 2 diabetes. Diabetes Care, v. 23, p. I563-80, 2000.

6. GENUTH, S. Lowering the criterion for impaired fasting glucose is in order. Diabetes Care, v. 26, n. 12, p. 3331-2, 2003.

7. GROSS, J.L. et al. Diabetes melito: diagnóstico, classificação e avaliação do controle glicêmico. Arq Bras Endocrinol Metab, v. 46, n. I, p. 16-26, 2002
8. GROSS, J.L.; FERREIRA, S.R.G.; OLIVEIRA, J.E. Glicemia pósprandial. Arq Bras Endocrinol Metab, v. 47, n. 6, p. 728-38, 2003.

9. HARRIS, M.I.; EASTMAN, R.C. Early detection of undiagnosed diabetes mellitus: a US perspective. Diabetes Metab Res Rer, v. 16, p. 230-6, 2000

10. KING, H.;AUBERT, R.E.; HERMAN,W. Global burden of diabetes, 1995-2025: prevalence, numerical estimates, and projections. Diabetes Care, v. 21 , p. 1414-31, 1998.

I I. MALERBI, D.A.; FRANCO, L.J. Multicenter study of the prevalence of diabetes mellitus and impaired glucose tolerance in the urban Brazilian population aged 30-69 yr: The Brazilian Cooperative Group on the Study of Diabetes Prevalence. Diabetes Care, v. I5, p. 1509-16, 1992.

12. NARAYAN, K.M. et al. Diabetes: a common, growing, serious, costly, and potentially preventable public health problem. Diabetes Res Clin Pract, v. 50, s. 2, p. S77-S84, 2000.

13. NATIONAL DIABETES DATA GROUP. Classification and diagnosis of diabetes mellitus and other categories of glucose intolerance. Diabetes, v. 28, p. 1039-57, 1979

I4. PASSOS,V.M.A. et al.Type 2 diabetes: prevalence and associated 
factors in a Brazilian community - the Bambuí health and aging study. São Paulo Med J, v. I23, n. 2, p. 66-7I, 2005.

15. SCHAAN, B.D'A.; HARZHEIM, E.; GUS, I. Perfil de risco cardíaco no diabetes mellitus e na glicemia de jejum alterada. Rev. Saúde Pública, v. 38, n. 4, p. 529-36, 2004.

16. SOCIEDADE BRASILEIRA DE DIABETES. Consenso Brasileiro sobre Diabetes: diagnóstico e classificação do diabetes melito e tratamento do diabetes melito do tipo 2. 2002. Disponível em: <http://www.diabetes.org.br. Acesso em: 21 abr 2005.

17. THE EXPERT COMMITTEE ON THE DIAGNOSIS AND CLASSIFICATION OF DIABETES MELLITUS. Report of the expert committee on the diagnosis and classification of diabetes mellitus. Diabetes Care, v. 20, p. I I83-97, 1997.

18. THE EXPERT COMMITTEE ON THE DIAGNOSIS AND
CLASSIFICATION OF DIABETES MELLITUS. Follow-up report on the diagnosis of diabetes mellitus. Diabetes Care, v. 26, n. II, p. 3160-7, 2003.

19.TORQUATO, M.T.C.G. et al. Prevalence of diabetes mellitus and impaired glucose tolerance in the urban population aged 30-69 years in Ribeirão Preto (São Paulo), Brazil. São Paulo Med J, v. I21, n. 6, p. 224-30, 2003.

20. TOSCANO, C.M. As campanhas nacionais para detecção das doenças crônicas não-transmissíveis: diabetes e hipertensão arterial. Ciência e Saúde Coletiva, v. 9, n. 4, p. 885-95, 2004.

21.WORLD HEALTH ORGANIZATION. Definition, diagnosis and classification of diabetes mellitus and its complications: report of a WHO consultation. Part I: Diagnosis and classifications of diabetes mellitus. World Health Organization, Geneva: WHO. 1999.59p. 